\title{
3 Research Soure \\ High Incidence of Postoperative Silent Venous Thromboembolism in Ulcerative Colitis
}

Norimitsu Shimada ( $\sim$ shimada@hiroshima-u.ac.jp )

Kure Medical Center

\section{Hiroki Ohge}

Hiroshima University Hospital

\section{Hiroki Kitagawa}

Hiroshima University Hospital

Kosuke Yoshimura

Hiroshima University Hospital

Norifumi Shigemoto

Hiroshima University Hospital

\section{Yusuke Watadani}

Hiroshima University Hospital

Shinnosuke Uegami

Hiroshima University Hospital

Kenichiro Uemura

Hiroshima University Hospital

\section{Shinya Takahashi}

Hiroshima University Hospital

\section{Research Article}

Keywords: ulcerative colitis, prophylaxis, D-dimer冈ileal pouch, portal-mesenteric venous thrombosis

Posted Date: January 12th, 2021

DOI: https://doi.org/10.21203/rs.3.rs-141629/v1

License: (c) (1) This work is licensed under a Creative Commons Attribution 4.0 International License.

Read Full License 


\section{Abstract}

Purpose: The incidence of postoperative venous thromboembolism (VTE) is high in patients with inflammatory bowel diseases. We aimed to analyze the incidence and predictive factors of postoperative VTE.

Methods: Patients with ulcerative colitis who underwent colon and rectum surgery during 2010-2018 were included. We retrospectively investigated the incidence of postoperative VTE.

Results: A total 140 cases of colorectal surgery were included. Postoperative VTE was detected in 24 (17.1\%). All patients with VTE had no specific symptoms related to thrombus, except one pulmonary embolism. Portal-mesenteric venous thrombosis was the most frequent $(18,75 \%)$; of these, 15 patients underwent total proctocolectomy (TPC) with ileal pouch-anal anastomosis (IPAA). In univariate analysis, VTE occurred more frequently in patients with neoplasia than refractory to medications $(27.2 \% \mathrm{vs} .12 .5 \%$; $p<0.031)$. TPC with IPAA was more associated with developing VTE $(28 \%)$ than total colectomy $(10.5 \%)$ or proctectomy (5.9\%). On logistic regression analysis of predictive risk factors, TPC with IPAA, total colectomy, longer operative time ( $>4$ hours), and high serum D-dimer levels $(>5.3 \mu \mathrm{g} / \mathrm{mL}$ ) the day following surgery were identified.

Conclusion: Postoperative VTE occurred frequently and asymptomatic, especially after TPC with IPAA. Ddimer the day after surgery may be useful predictor of VTE.

\section{Introduction}

Venous thromboembolism (VTE) is a well-known complication after colorectal surgery, which causes morbidity and mortality in hospitalized as well as discharged patients. In particular, patients with ulcerative colitis (UC) show a higher incidence of VTE postoperatively.

Current guidelines from the American Society of Colon and Rectal Surgeons recommend pharmacological prophylaxis with low-dose unfractionated heparin or low-molecular-weight heparin in patients with moderate and high risk following abdominal surgery. Patients with inflammatory bowel disease (IBD) are defined as having a high risk for VTE [1].

Despite many reports on postoperative VTE and guidelines recommending pharmacological prophylaxis, this is not sufficiently administered in clinical practice. There might be some reasons, the risk of postoperative bleeding from remnant rectum with acute flare, epidural hematoma, and mostly surgeon's low recognition of postoperative VTE.

Many previous studies on VTE have been conducted in Western countries, and these have reported that the risk of postoperative VTE is 2- to 3-fold higher in patients with UC, with rates of VTE of $4.1-5.8 \%$ [2] [3]. 
We suspect that more postoperative VTE occur in patients with UC in our clinical setting than the findings of previous reports. As the data in past studies were extracted from large nationwide databases, these are considered reliable and there is less likelihood of bias in such large datasets. However, it is possible that only patients with symptomatic VTE were registered and that many patients with asymptomatic VTE were not included in those data. We therefore conducted a retrospective study of postoperative VTE in patients with UC in IBD-specialized facilities of Japan and reviewed all computed tomography (CT) scans that had been examined postoperatively. The aim of this study was to clarify the incidence of postoperative VTE including the asymptomatic thrombus and to identify predictive factors for VTE.

\section{Materials And Methods}

\section{Data collection and variables}

We performed this retrospective observational study using a prospectively maintained database of patients with UC undergoing abdominopelvic bowel resection at Hiroshima University Hospital between January 2010 and December 2018. In total, we included 143 operation cases among 110 patients. Among these cases, we excluded three cases of total colectomy (TC) with preoperative VTE (Fig. 1). Restorative proctocolectomy and proctectomy were conducted with rectal mucosectomy and hand-sewn pouch-anal anastomosis with a J pouch. We reviewed patients' electronic medical charts to obtain information regarding patient characteristics, surgical procedures, and postoperative data. Emergency surgery was defined an unplanned surgery on admission. The preoperative state of venous thrombosis was accessed using contrast-enhanced $\mathrm{CT}$, the image range was from chest to pelvic, just prior to emergency surgery, and within at least 4 weeks of elective surgery. All patients underwent early mobilization on the day following surgery and used mechanical VTE prophylaxis, with compression stockings and an intermittent pneumatic compression device, from the time of surgery until the start of walking. As pharmacological prophylaxis, low molecular heparin or unfractionated heparin had used for 7days after surgery, and administration was decided by surgeons.

\section{Outcome}

The primary outcome was incidence of in-hospital VTE after abdominopelvic surgery. The secondary outcome was predictive risk factors for venous thrombosis.

In this study, VTE included pulmonary embolism (PE), deep vein thrombosis (DVT), and portalmesenteric venous thrombosis (PMVT). DVT included thrombosis at the vena cava, internal jugular vein, subclavian vein, and femoral vein. Diagnosis of VTE was assessed using contrast-enhanced CT examination as well as supplementary ultrasound examination. The preoperative therapy included use of steroid just prior to surgery, immunosuppressive agents within 1 week before surgery, and biological agents within 6 weeks prior to surgery. 


\section{Statistical analysis}

Analysis was conducted with JMP version 11 (SAS Institute Japan Ltd., Tokyo, Japan). Statistics are reported as median with interquartile range (IQR) for continuous variables and frequency with percentage for categorical variables. Normally distributed continuous variables were analyzed using the $t$-test, and nonnormally distributed continuous variables were analyzed with the nonparametric Wilcoxon's rank-sum test. Fisher's exact test was performed for categorical variables. Multivariable logistic regression analysis was conducted to identify predictive risk factors for surgical recurrence at the anastomosis site. Each analysis was performed using a two-sided $5 \%$ significance level and $95 \%$ confidence interval.

\section{Ethical considerations}

This study was approved by the Hiroshima University institutional review board (E-1636). Participants received information about the conduct of the research, including the purpose of the study, and were given the opportunity to decline participation in the study. Because the opt-out method can be used without informed consent from the patient, it was published on the web.

\section{Results}

We included a total 110 patients with UC in this study. The total number of operations was 143 cases, including 64 cases of TPC with IPAA, 40 cases of TC with ileostomy, 34 cases of proctectomy with IPAA, 4 cases of partial colectomy, and one case of TC with ileorectal anastomosis. Three patients with TC who had preoperative VTE were excluded from the analysis (Fig. 1). Of these 140 cases, postoperative VTE was detected in 24 cases $(17.1 \%)$ at 7 (5.2-9.7) days after surgery.

Contrast-enhanced CT after surgery was conducted in 91 cases (65\%). The most frequent reason for CT examination were suspicion of pelvic abscess or outlet obstruction at the stoma site with elevated fever, abdominal pain, and ileus. Eighteen cases (12.8\%) with elevated serum D-dimer levels or transaminase were suspected of VTE prior to CT examination, and VTE was detected in 5 cases of those. All patients with VTE after surgery had no specific symptoms related to VTE, except for one patient who had pulmonary embolism with dyspnea on walking.

Regarding the site of thromboembolism, PMVT occurred most frequently (18 patients, 75\%). Of those, 15 patients were detected after TPC + IPAA (Fig. 2). All 17 cases of liver portal vein thrombosis were detected in the right hepatic lobe, and 5 of these cases showed mesenteric venous thrombosis simultaneously. With respect to mesenteric venous thrombosis, five cases were in the inferior mesenteric vein, 2 cases in the superior mesenteric vein, and one case was in the splenic vein. PE occurred in four cases $(2.8 \%$, three cases after TPC + IPAA, and one case after TC). One subclavian vein thrombus and one jugular vein thrombus was detected after TC. 
The rate of postoperative VTE prophylaxis with anticoagulant was $31.4 \%$ in all patients, $41.7 \%$ in the VTE (+) group and $29.3 \%$ in the VTE (-) group, respectively. The surgical procedure was TPC + IPAA 28 cases (43.7\%), TC in 6 cases (16.2\%), and proctectomy with IPAA in 10 cases $(29.4 \%)$.

On univariate analysis, VTE occurred more frequently in patients with neoplasia (cancer or high-grade dysplasia) than refractory to medications $(27.2 \%$ vs. $12.5 \% ; p<0.031)$. There was no difference in age, body mass index, American Society of Anesthesiology-physical status (ASA-PS) score $\geq 3$, current smoking, preoperative therapy, and comorbidity between the VTE (+) group and VTE (-) group (Table 1). The perioperative results showed significant differences in the rates of VTE according to surgical procedure. Patients who underwent TPC with IPAA were more likely to develop VTE (28\%) than TC $(10.5 \%)$ or proctectomy $(5.9 \%)$. Operative time in the VTE $(+)$ group was significantly longer than in the VTE (-) group (340 vs. 291 minutes, $p=0.006$ ). Laboratory data indicated that serum D-dimer levels on the day following surgery were higher in the VTE $(+)$ group $(5.6 \mu \mathrm{g} / \mathrm{mL}$ vs. $3.1 \mu \mathrm{g} / \mathrm{mL}, p=0.00218)$. Ddimer at day 3 after surgery was also higher in the VTE (+) group, but this was not significantly different (Table 2). 
Table 1

Patient characteristics according to venous thromboembolism

\begin{tabular}{|c|c|c|c|c|}
\hline & overall & VTE + & VTE - & $p$ value \\
\hline & $N=140$ & $N=24$ & $N=116$ & \\
\hline \multicolumn{5}{|l|}{ Gender, n (\%) } \\
\hline Male & $97(69.3)$ & $15(62.5)$ & $82(70.6)$ & 0.428 \\
\hline Female & $43(31.7)$ & $9(37.5)$ & $34(29.3)$ & \\
\hline Age, median (range) & $54(41.2-63)$ & $48(38.2-57.5)$ & $55.5(42-64.7)$ & 0.158 \\
\hline BMI, median (range) & $21(18.7-24.1)$ & $21.8(18.4-24)$ & $20.5(18.7-24.1)$ & 0.81 \\
\hline ASA-PS $\geqq 3, n(\%)$ & $8(5.7)$ & $1(4.2)$ & $7(6.0)$ & 0.719 \\
\hline Current smoking, n (\%) & $13(9.3)$ & $2(8.3)$ & $11(9.48)$ & 0.86 \\
\hline \multicolumn{5}{|l|}{ Preoperative therapy, n (\%) } \\
\hline Steroid & $59(42.1)$ & $13(54.2)$ & $46(39.6)$ & 0.19 \\
\hline Immunosuppressive agents & $48(34.2)$ & $9(37.5)$ & $39(33.6)$ & 0.72 \\
\hline Biological agents & $20(14.2)$ & $3(12.5)$ & $17(14.7)$ & 0.78 \\
\hline \multicolumn{5}{|l|}{ Comorbidity, n (\%) } \\
\hline HT & $20(14.2)$ & $5(20.8)$ & $15(8.6)$ & 0.31 \\
\hline $\mathrm{HL}$ & $11(7.9)$ & $1(4.2)$ & $10(8.6)$ & 0.46 \\
\hline DM & $15(10.7)$ & 0 & $15(12.9)$ & 0.073 \\
\hline \multicolumn{5}{|l|}{ Indication, n (\%) } \\
\hline cancer/ dysplasia & $44(31.4)$ & $12(50)$ & $32(27.5)$ & \\
\hline refractory to medication & $96(68.5)$ & $12(50)$ & $84(87.5)$ & $\star 0.0313$ \\
\hline
\end{tabular}


Table 2

Peri-operative results according to venous thromboembolism

\begin{tabular}{|c|c|c|c|c|}
\hline & overall & VTE + & VTE - & $p$ value \\
\hline & $N=140$ & $N=24$ & $N=116$ & \\
\hline \multicolumn{5}{|l|}{ Procedure } \\
\hline TPC $\square$ IPAA, n (\%) & $64(45.7)$ & 18(75) & $46(39.7)$ & \\
\hline TC, n (\%) & $38(27.1)$ & $4(16.7)$ & $34(29.3)$ & \\
\hline Proctectomy + IPAA, n (\%) & $34(24.2)$ & $2(8.3)$ & $32(27.6)$ & \\
\hline Other, n (\%) & $4(2.8)$ & 0 & $4(3.4)$ & *0.0144 \\
\hline IPAA & $96(68.6)$ & $20(83.3)$ & $76(65.5)$ & 0.087 \\
\hline Operative time, min.(range) & $\begin{array}{l}291(203- \\
383)\end{array}$ & $\begin{array}{l}340(255- \\
476)\end{array}$ & $\begin{array}{l}277(181- \\
355)\end{array}$ & $\star 0.006$ \\
\hline Blood loss, ml (range) & $\begin{array}{l}300(155- \\
522)\end{array}$ & $\begin{array}{l}326(230- \\
528)\end{array}$ & $275(12-520)$ & 0.1775 \\
\hline \multicolumn{5}{|l|}{ Complications } \\
\hline Ileus, n (\%) & $28(20)$ & $6(25)$ & $22(19)$ & 0.57 \\
\hline Anastomosis leakage, n (\%) & $9(6.4)$ & $1(4.2)$ & $8(6.9)$ & 0.629 \\
\hline Pelvic abscess, n (\%) & $24(17)$ & $3(12.5)$ & $21(18)$ & 0.497 \\
\hline Emergency operation & $36(27.6)$ & $5(20.8)$ & $31(26.7)$ & 0.5478 \\
\hline $\begin{array}{l}\text { Prophylaxis with anticoagulant, } \mathrm{n} \\
(\%)\end{array}$ & $44(31.4)$ & $10(41.7)$ & $34(29.3 \%)$ & 0.2956 \\
\hline \multicolumn{5}{|l|}{ D-dimer, $\mu \mathrm{g} / \mathrm{ml}$ (range) } \\
\hline POD1 D-dimer, & $3.1(2.2-5.8)$ & $5.6(2.7-8.9)$ & $3(2.2-4.7)$ & *0.0218 \\
\hline POD3 D-dimer & $4.4(3.1-8.25)$ & $7(3.5-10.6)$ & $4.1(3.1-7.7)$ & 0.083 \\
\hline Peak D-dimer & $8.85(5.9-13)$ & $\begin{array}{l}10.1(7.4- \\
15.3)\end{array}$ & $8.7(5.7-12)$ & 0.072 \\
\hline
\end{tabular}

In logistic regression analysis, we identified that TPC with IPAA, TC, longer operative time ( $>4$ hours), and serum D-dimer level $>5.3 \mathrm{mg} / \mathrm{dL}$ on the day following surgery were predictive risk factors for postoperative VTE in patients with UC (Table 3 ). 
Table 3

Predictive risk factors in logistic regression analysis

\begin{tabular}{|lllll|}
\hline & & OR & $95 \% \mathrm{Cl}$ & $p$ value \\
\hline Procedure & Proctectomy + IPAA & reference & - & - \\
\hline & TC & 45.88 & $1.44-2863$ & $* 0.0304$ \\
\hline TPC + IPAA & 17.12 & $1.675-433.7$ & $* 0.015$ \\
\hline Ondication & cancer/dysplasia & 0.45 & $0.06-2.89$ & 0.405 \\
\hline $\begin{array}{l}\text { D-dimerative time } \\
\text { Abbreviations: OR, odds ratio; Cl, confidence interval; TPC, total proctocolectomy; IPAA, ileal pouch- } \\
\text { anal anastomosis; TC, total colectomy. }\end{array}$ & $(>4 \mathrm{~h})$ & 28.84 & $1.23-1260$ & $* 0.0358$ \\
\hline
\end{tabular}

\section{Discussion}

In this study, postoperative VTE in patients with UC was detected in 24 cases (17.1\%) at a median 7 days after surgery. PMVT was the most common site of VTE (18 cases,12.8\%), and that was occurred frequently after TPC with IPAA (23.4\%). Three predictive risk factors detected in logistic regression analysis (Table 3 ) were elevated D-dimer on the day after surgery, surgical procedure (TPC with IPAA, TC) and long operation time (over 4 hours).

IBD is a well-known independent risk factor for VTE, and patients with UC have higher risk of VTE than those with Crohn's disease [3] [4]. In patients with IBD, the risk of developing DVT and PE was 1.98- and 1.80-fold higher than other individuals, and the rates of postoperative VTE were $2.5-3.8 \%$, which was higher than the $2.4 \%$ among patients with colorectal cancer [5-9]. The incidence rate of VTE in our study $(17.1 \%)$ was higher than previous reports. One of the reasons is that our study was including asymptomatic VTE. CT examination after surgery was conducted for 91 cases (65\%), and many asymptomatic venous thrombus were coincidentally identified. It's only 5 cases those were suspected of VTE before CT, and other 19 cases were coincident. Frequent postoperative CT might be related with Universal Insurance System for all people in Japan, which allow us to use informative CT easily in order to detect the postoperative complications.

PMVT was frequently detected after TPC + IPAA in our study. There are few studies regarding PMVT. The reported rate of symptomatic PMVT after colorectal surgery is $2.9-4.9 \%[10,11]$ [12]. IBD is a frequent cause of PMVT after abdominal surgery, with a reported rate of $8.3 \%$ [13]. As a surgical procedure, restorative TPC with IPAA for patients with UC has been reported as an independent predictor of PMVT, and the incidence rate was as high as $5.8-10 \%[14][11,15][16]$. In the procedure of ileal pouch construction and anal anastomosis, there were several factors to promote thrombus in addition to the nature of IBD. Those were long operation time, pelvic surgery in the lithotomy position, manipulation of 
mesenteric vessels, and traction of the superior mesentery vessels related to IPAA reconstruction. Dehydrated state caused by covering ileostomy was also related to thrombosis, which was created for every patient at our institution.

D-dimer elevation (>5.3 $\mathrm{mg} / \mathrm{dL}$ ) a day after surgery was detected as predictive risk factor for postoperative VTE in this study, and the sensitivity was $55 \%$, and the specificity was $78.5 \%$. D-dimer is not specific marker only for thrombus. Disease flares, bleeding, surgical invasion or a septic condition are also related to D-dimer elevation, even though they didn't have VTE. Actually, 5 cases in VTE negative group showed very high D-dimer levels $(>10 \mu \mathrm{g} / \mathrm{mL}$ ), all of them were underwent emergent TC for bleeding or perforation with severe bowel inflammation. Therefore, D-dimer elevation isn't strong predictor of VTE, but we can use easily to suspect the thrombus. It's may be more useful for patients undergoing TPC or proctectomy whose preoperative condition is relatively stable.

This study had several limitations. First, the study was designed retrospectively at a single institution. Variables that could be collected from the database were limited. Pharmacological prophylaxis was not administered to all patients, and the administration period was not defined. CT examination was conducted in all patients preoperatively but not in all patients following surgery, and not exam the lower limb venous thrombus. Data of lower limb examination using ultrasound imaging were not collected. Lastly, the percentage of postoperative VTE prophylaxis with anticoagulant was only $31.4 \%$ in this study. This is too small in spite of that the risks of VTE in patients with UC has well known. Guideline states that strict administration of postoperative pharmacological prophylaxis was necessary, except the case having the risk of bleeding from remnant rectum [1].

\section{Conclusion}

We detected more postoperative VTE in our retrospective dataset than previous data. Especially, quarter cases of TPC + IPAA showed PMVT. Most cases were asymptomatic VTE but had the potential to be symptomatic with growth. It's important to recognize this result, and conduct the strict anticoagulant therapy.

\section{Abbreviations}

VTE: venous thromboembolism, TPC: total proctocolectomy, IPAA: ileal pouch-anal anastomosis, UC: ulcerative colitis, IBD; inflammatory bowel disease, CT: computed tomography, TC: total colectomy, PE: pulmonary embolism, DVT: deep vein thrombosis, PMVT: portal-mesenteric venous thrombosis, IQR: interquartile range,

\section{Declarations}

\section{Ethics approval and consent to participate}


All mehods were performed in accordance with the Declaration of Helsinki. This study was approved by the Hiroshima University institutional review board (E-1636). The need of informed consent is waived by Hiroshima University hospital IRB. All participants received information about the conduct of the research, including the purpose of the study, and were given the opportunity to decline participation in the study. Because the opt-out method can be used without informed consent from the patient who had finished the surgery, it was published on the web.

\section{Consent for publications}

Not applicable

\section{Availability of data and materials}

The database used and analysed during the current study are available from the corresponding author on reasonable request.

\section{Competing interest}

The authors declare that they are no competing interests.

\section{Funding}

This study has never funded by any foundation, company, or external source.

\section{Author's Contributions.}

NShima, NShige, and HO made the concept of this study. NShima, and YW design this study. HK, KY, and NShima collected data. ST, KU analyzed data and interpretated. Manuscript was prepared by NShima and SU, and edited by $\mathrm{HO}$ and ST. All authors read and approved the final manuscript.

\section{Acknowledgments}

We thank Analisa Avila, ELS, of Edanz Group (www.edanzediting.com/ac) for editing a draft of this manuscript.

\section{References}


1. Fleming F, Gaertner W, Ternent CA, Finlayson E, Herzig D, Paquette IM, et al. The American Society of Colon and Rectal Surgeons Clinical Practice Guideline for the Prevention of Venous Thromboembolic Disease in Colorectal Surgery. Diseases of the colon and rectum. 2018;61:14-20.

2. Ali F, Al-Kindi SG, Blank JJ, Peterson CY, Ludwig KA, Ridolfi TJ. Elevated Venous Thromboembolism Risk Following Colectomy for IBD Is Equal to Those for Colorectal Cancer for Ninety Days After Surgery. Diseases of the colon and rectum. 2018;61:375-81.

3. Brady MT, Patts GJ, Rosen A, Kasotakis G, Siracuse JJ, Sachs T, et al. Postoperative Venous Thromboembolism in Patients Undergoing Abdominal Surgery for IBD: A Common but Rarely Addressed Problem. Diseases of the colon and rectum. 2017;60:61-7.

4. Wallaert JB, De Martino RR, Marsicovetere PS, Goodney PP, Finlayson SRG, Murray JJ, et al. Venous thromboembolism after surgery for inflammatory bowel disease: are there modifiable risk factors? Data from ACS NSQIP. Diseases of the colon and rectum. 2012;55:1138-44.

5. Chung WS, Lin CL, Hsu WH, Kao CH. Inflammatory bowel disease increases the risks of deep vein thrombosis and pulmonary embolism in the hospitalized patients: a nationwide cohort study. Thromb Res. 2015;135:492-6.

6. Gross ME, Vogler SA, Mone MC, Sheng X, Sklow B. The importance of extended postoperative venous thromboembolism prophylaxis in IBD: a National Surgical Quality Improvement Program analysis. Diseases of the colon and rectum. 2014;57:482-9.

7. Benlice C, Holubar SD, Gorgun E, Stocchi L, Lipman JM, Kalady MF, et al. Extended Venous Thromboembolism Prophylaxis After Elective Surgery for IBD Patients: Nomogram-Based Risk Assessment and Prediction from Nationwide Cohort. Diseases of the colon and rectum. 2018;61:1170-9.

8. McKenna NP, Behm KT, Ubl DS, Glasgow AE, Mathis KL, Pemberton JH, et al. Analysis of Postoperative Venous Thromboembolism in Patients With Chronic Ulcerative Colitis: Is It the Disease or the Operation? Diseases of the colon and rectum. 2017;60:714-22.

9. Wilson MZ, Connelly TM, Tinsley A, Hollenbeak CS, Koltun WA, Messaris E. Ulcerative Colitis Is Associated With an Increased Risk of Venous Thromboembolism in the Postoperative Period: The Results of a Matched Cohort Analysis. Annals of surgery. 2015;261:1160-6.

10. Robinson KA, O'Donnell ME, Pearson D, Kriegshauser JS, Odeleye M, Kalkbrenner K, et al. Portomesenteric venous thrombosis following major colon and rectal surgery: incidence and risk factors. Surgical endoscopy. 2015;29:1071-9.

11. Allaix ME, Krane MK, Zoccali M, Umanskiy K, Hurst R, Fichera A. Postoperative portomesenteric venous thrombosis: lessons learned from 1,069 consecutive laparoscopic colorectal resections. World journal of surgery. 2014;38:976-84.

12. Erem HH, Kiran RP, Remzi FH, Vogel JD. Venous thromboembolism in colorectal surgery: skip SCIP or comply? Techniques in coloproctology. 2014;18:719-24.

13. Kayal M, Radcliffe M, Plietz M, Rosman A, Greenstein A, Khaitov S, et al. Portomesenteric Venous Thrombosis in Patients Undergoing Surgery for Medically Refractory Ulcerative Colitis. Inflammatory 
bowel diseases. 2020;26:283-8.

14. Gu J, Stocchi L, Gorgun E, Remzi FH. Risk factors associated with portomesenteric venous thrombosis in patients undergoing restorative proctocolectomy for medically refractory ulcerative colitis. Colorectal disease : the official journal of the Association of Coloproctology of Great Britain and Ireland. 2016;18:393-9.

15. Remzi FH, Fazio VW, Oncel M, Baker ME, Church JM, Ooi BS, et al. Portal vein thrombi after restorative proctocolectomy. Surgery. 2002;132:655-62.

16. Ball CG, MacLean AR, Buie WD, Smith DF, Raber EL. Portal vein thrombi after ileal pouch-anal anastomosis: its incidence and association with pouchitis. Surgery today. 2007;37:552-7.

\section{Figures}

Fig. 1

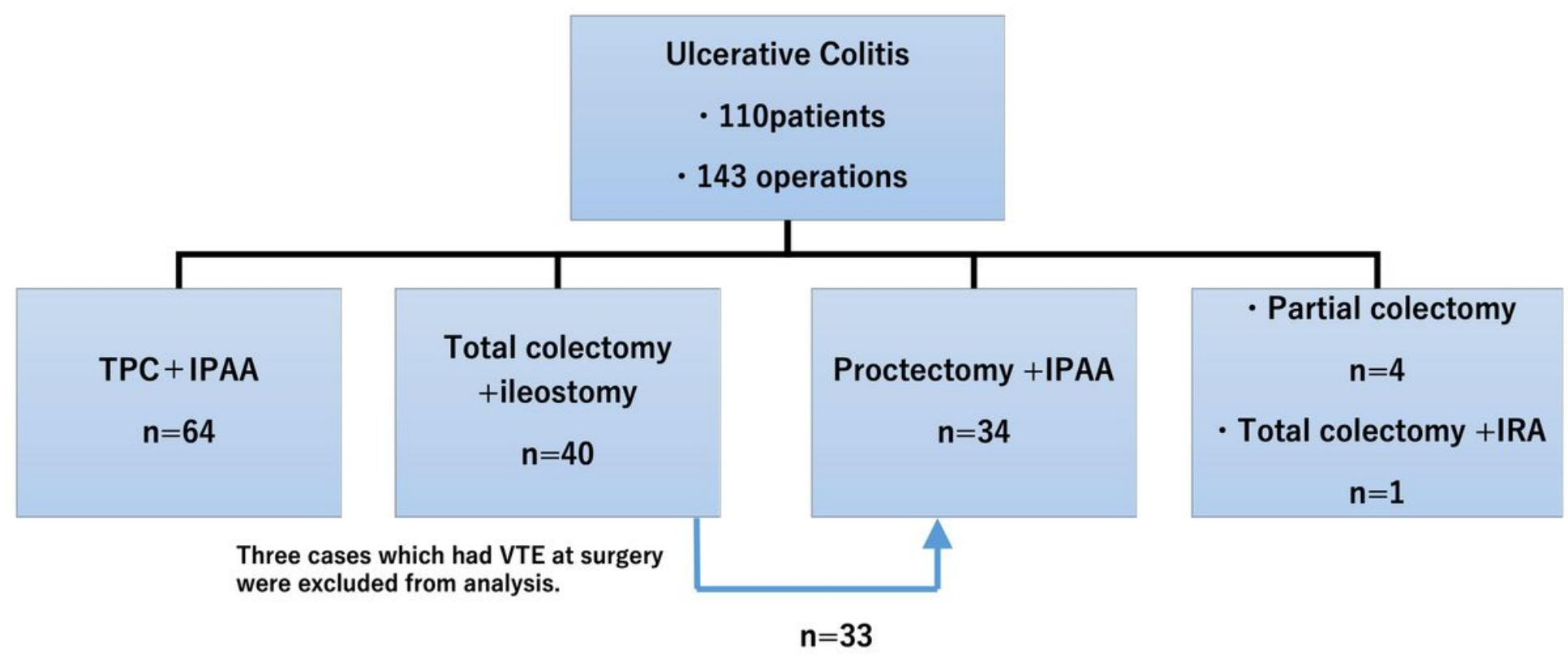

Figure 1

We enrolled a total 110 patients, including 143 operations. After total colectomy, 33 patients underwent proctocolectomy with IPAA. In total colectomy, 3 cases were excluded from the analysis because they had preoperative venous thromboembolism. IPAA, ileal pouch-anal anastomosis. 
Fig. 2

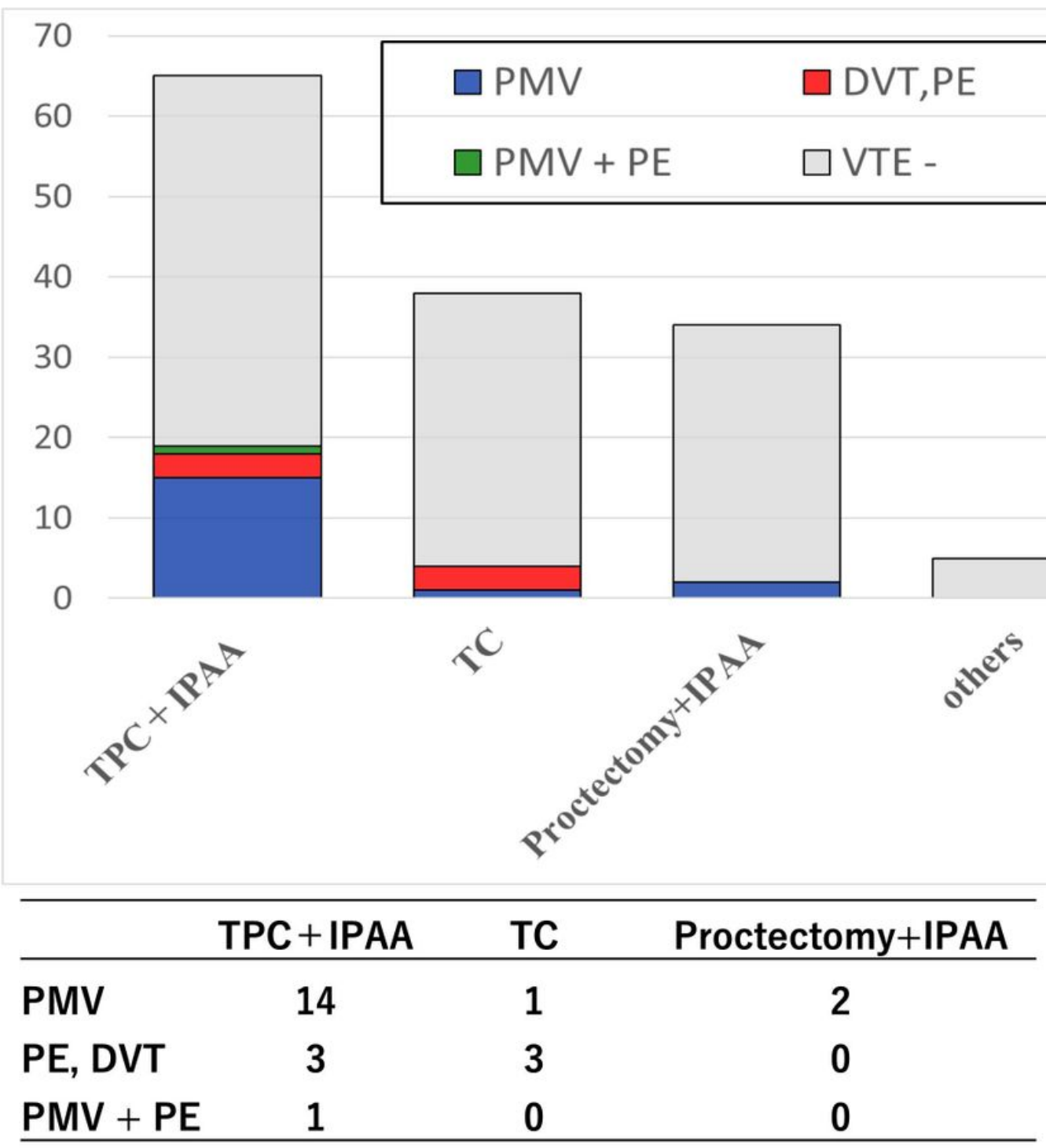

Figure 2

Correlation between surgical procedure and thrombosis site. PMVT frequently occurred after TPC with IPAA. PE and DVT occurred more frequently in patients who underwent TC with acute flare-ups of UC. DVT was included the central vein and femoral vein thrombus. TPC, total proctocolectomy; IPAA, ileal pouch-anal anastomosis; TC, total colectomy; PMV, portal-mesenteric venous thrombosis. 